\title{
Structural and biochemical characterization of
}

\section{the interaction of tubulin with potent natural}

\section{analogs of podophyllotoxin}

${ }^{\dagger}$ Mayra Antunez, ${ }^{\dagger}$ Javier Rodríguez-Salarichs, ${ }^{\star}$ Mariano Redondo-Horcajo, ${ }^{\dagger}$ Alejandra León, ${ }^{\star}$ Isabel Barasoain, ${ }^{\S}$ Ángeles Canales, ${ }^{\star}$ F. J. Cañada, ${ }^{\perp J}$ Jesús Jiménez-Barbero, ${ }^{\dagger}$ Laura Álvarez* ${ }^{\ddagger}$ J. Fernando Díaz*

${ }^{\dagger}$ Centro de Investigaciones Químicas-IICBA, Universidad Autónoma del Estado de

Morelos, Cuernavaca, Morelos 62209, México

${ }^{\S}$ Departamento de Química Orgánica I, Facultad Ciencias Químicas, Universidad

Complutense de Madrid, Avenida Complutense s/n, 28040 Madrid, Spain

${ }^{t}$ Department of Chemical and Physical Biology, Centro de Investigaciones Biológicas, Consejo Superior de Investigaciones Científicas, Ramiro de Maeztu 9, 28040 Madrid, Spain

${ }^{\perp}$ CIC bioGUNE Parque Tecnológico de Bizkaia, Edif. 801A-1º, 48160 Derio- Bizkaia, Spain and Ikerbasque, Basque Foundation for Science, Maria Diaz de Haro 3, 48009 Bilbao, Spain 


\section{List of Supplementary information.}

Supplementary Table 01. Cell Cycle Distribution of A549 Cells Treated with 1-5 as compared to Taxol.

Supplementary Table 02. Inhibition of Tubulin Assembly by the Natural Lignans. Pelleted tubulin $(\mu \mathrm{M})$ of $\mathbf{1 - 5}$.

Supplementary Table 03. NMR Parameters (Chemical Shifts, $\delta$ (ppm) and Multiplicity) for $\mathbf{1}, \mathbf{2}$, and $\mathbf{3}$ at $298 \mathrm{~K}$ in $\mathrm{D}_{2} \mathrm{O}$.

S.1. STD-NMR (600 MHz, $\left.\mathrm{D}_{2} \mathrm{O}\right)$ spectrum of compound $\mathbf{1}$.

S.2. STD-NMR (600 MHz, $\left.\mathrm{D}_{2} \mathrm{O}\right)$ spectrum of compound 2.

S.3. STD-NMR (600 MHz, $\left.\mathrm{D}_{2} \mathrm{O}\right)$ spectrum of compound 3.

S.4. TR-NOESY $\left(600 \mathrm{MHz}, \mathrm{D}_{2} \mathrm{O}\right)$ spectrum of compound $\mathbf{3}$ in free state.

S.5. TR-NOESY (600 MHz, $\left.\mathrm{D}_{2} \mathrm{O}\right)$ spectrum of compound 3 in complex state with dimer of tubulin. 
Supplementary Table 01. Percentage of Cell Cycle Distribution of A549 Cells Treated with 1-5 as Compared to Taxol

\begin{tabular}{ccccc}
\hline Compound & $\begin{array}{c}\text { Sub G1 } \\
\text { phase }\end{array}$ & $\begin{array}{c}\text { G0/G1 } \\
\text { phase }\end{array}$ & S phase & G2/M phase \\
\hline DMSO & 2 & 78 & 7 & 13 \\
Taxol (50nM) & 6 & 16 & 8 & 70 \\
$\mathbf{1}(\mathbf{1 0 0 n M})$ & 2 & 19 & 6 & 73 \\
$\mathbf{2}(\mathbf{1 0 0 n M})$ & 1 & 14 & 6 & 79 \\
$\mathbf{3}(\mathbf{1 0 0} \mathbf{n})$ & 5 & 16 & 6 & 73 \\
$\mathbf{4}(\mathbf{1} \boldsymbol{\mu M})$ & 2 & 22 & 7 & 69 \\
$\mathbf{5}(\mathbf{2 5} \boldsymbol{\mu M})$ & 4 & 28 & 7 & 61 \\
\hline
\end{tabular}

${ }^{\mathrm{a}}$ Cells were incubated for 20 hours with several dilutions of each ligand. Numbers in the table are percentages (\%) of cells in each phase of the cell cycle. The sub G1 peaks are presumably apoptotic cells.

Supplementary Table 02. Percentage of the Inhibition of Tubulin Assembly by the Natural Lignans. Pelleted Tubulin $(\mu \mathrm{M})$

\begin{tabular}{|c|c|c|c|c|c|}
\hline$\mu \mathrm{M}$ & 1 & 2 & 3 & 4 & 5 \\
\hline 0 & $19.0 \pm 2.19$ & $\begin{array}{l}24.53 \pm \\
2.82\end{array}$ & $\begin{array}{l}23.87 \pm \\
3.76\end{array}$ & $\begin{array}{l}21.23 \pm \\
3.23\end{array}$ & $\begin{array}{l}18.08 \pm \\
2.09\end{array}$ \\
\hline 0.1 & $23.17 \pm 1.45$ & $\begin{array}{l}25.58 \pm \\
0.98\end{array}$ & $\begin{array}{l}25.70 \pm \\
3.34\end{array}$ & $\begin{array}{l}24.15 \pm \\
0.32\end{array}$ & $\begin{array}{l}24.12 \pm \\
0.86\end{array}$ \\
\hline 0.5 & $\begin{array}{l}21.367 \pm \\
1.19\end{array}$ & $\begin{array}{l}24.62 \pm \\
1.65\end{array}$ & $\begin{array}{l}22.84 \pm \\
3.40\end{array}$ & $\begin{array}{l}25.76 \pm \\
2.00\end{array}$ & $\begin{array}{l}22.84 \pm \\
0.84\end{array}$ \\
\hline 1 & $22.97 \pm 1.91$ & $\begin{array}{l}19.69 \pm \\
2.12\end{array}$ & $\begin{array}{l}25.92 \pm \\
1.50\end{array}$ & $\begin{array}{l}23.66 \pm \\
1.88\end{array}$ & $\begin{array}{l}24.83 \pm \\
0.75\end{array}$ \\
\hline 5 & $15.08 \pm 3.59$ & $\begin{array}{l}10.05 \pm \\
4.19\end{array}$ & $\begin{array}{l}25.51 \pm \\
2.26\end{array}$ & $\begin{array}{l}18.62 \pm \\
7.51\end{array}$ & $\begin{array}{l}24.15 \pm \\
0.31\end{array}$ \\
\hline 10 & $6.36 \pm 2.87$ & $\begin{array}{l}3.53 \pm 1.57 \\
3.22 \pm 1.32\end{array}$ & $\begin{array}{l}24.46 \pm \\
2.87 \\
13.53 \pm \\
0.30\end{array}$ & $\begin{array}{l}20.82 \pm \\
2.05 \\
12.13 \pm \\
3.33\end{array}$ & $\begin{array}{l}24.12 \pm \\
1.20 \\
24.52 \pm \\
0.72\end{array}$ \\
\hline
\end{tabular}


Supplementary Table 03. NMR Parameters (Chemical Shifts, $\delta$ in ppm and Multiplicity) for 1, 2, and 3 at $298 \mathrm{~K}$ in $\mathrm{D}_{2} \mathrm{O}$

\begin{tabular}{|c|c|c|c|}
\hline Proton & $\begin{array}{c}1 \\
\delta_{\mathrm{H}} \text { mult }(J, \mathrm{~Hz})\end{array}$ & $\stackrel{2}{2} \delta_{\mathrm{H}}$ mult $(J, \mathrm{~Hz})$ & $\begin{array}{c}3 \\
\delta_{\mathrm{H}} \operatorname{mult}(J, \mathrm{~Hz}) \\
\end{array}$ \\
\hline H-6 & $7.09, s$ & $6.93, s$ & --- \\
\hline H-5' & --- & --- & $6.78, d(8.5)$ \\
\hline H-2' & $6.44, s$ & $6.46, s$ & $6.76, d(1.5)$ \\
\hline H-6' & $6.44, s$ & $6.46, s$ & $\begin{array}{c}6.46, d d(1.4 \\
7.27)\end{array}$ \\
\hline H-3 & $6.49, s$ & $6.56, s$ & $6.23, s$ \\
\hline $\mathrm{O}-\mathrm{CH}_{2}-\mathrm{O}$ & $5.9, s$ & $5.96, s$ & $5.8, s$ \\
\hline H-7' & $4.58, m$ & $4.57^{\mathrm{a}}$ & $4.5, m^{\mathrm{a}}$ \\
\hline H-9 $\alpha$ & $4.58, m$ & $4.42, m$ & $4.41, m$ \\
\hline H-9 $\beta$ & $4.2, m$ & $4.26, m$ & $3.96, m$ \\
\hline $\mathrm{OCH}_{3}-6$ & --- & -- & $3.85, s$ \\
\hline $\mathrm{OCH}_{3}-4^{\prime}$ & $3.67, s$ & $3.68, s$ & $3.64, s$ \\
\hline $\mathrm{OCH}_{3}-5^{\prime}$ & $3.7, s$ & $3.71, s$ & --- \\
\hline $\mathrm{OCH}_{3}-3^{\prime}$ & $3.7, s$ & $3.71, s$ & $3.66, s$ \\
\hline H-7 $\alpha$ & --- & --- & $\begin{array}{c}3.07, d d(5.15, \\
15.8)\end{array}$ \\
\hline $\mathrm{H}-8^{\prime} \beta$ & $\begin{array}{c}3.19, d d(5.5, \\
13.9)\end{array}$ & $\begin{array}{c}3.32, d d(4.9 \\
14.7)\end{array}$ & $2.87, m$ \\
\hline H-8 $\alpha$ & $2.80, m$ & $2.89, m$ & $2.61, m$ \\
\hline $\mathrm{H}-7 \beta$ & $4.7, d^{\mathrm{a}}$ & $5.84, d(6.3)$ & $2.41, m$ \\
\hline OAc & --- & $2.15, s$ & --- \\
\hline
\end{tabular}

${ }^{\mathrm{a}}$ Water suppression region, $10 \mathrm{mM}$ sodium phosphate, $0.1 \mathrm{mM}$ GTP, $\mathrm{D}_{2} \mathrm{O}$. 
STD Spectra.

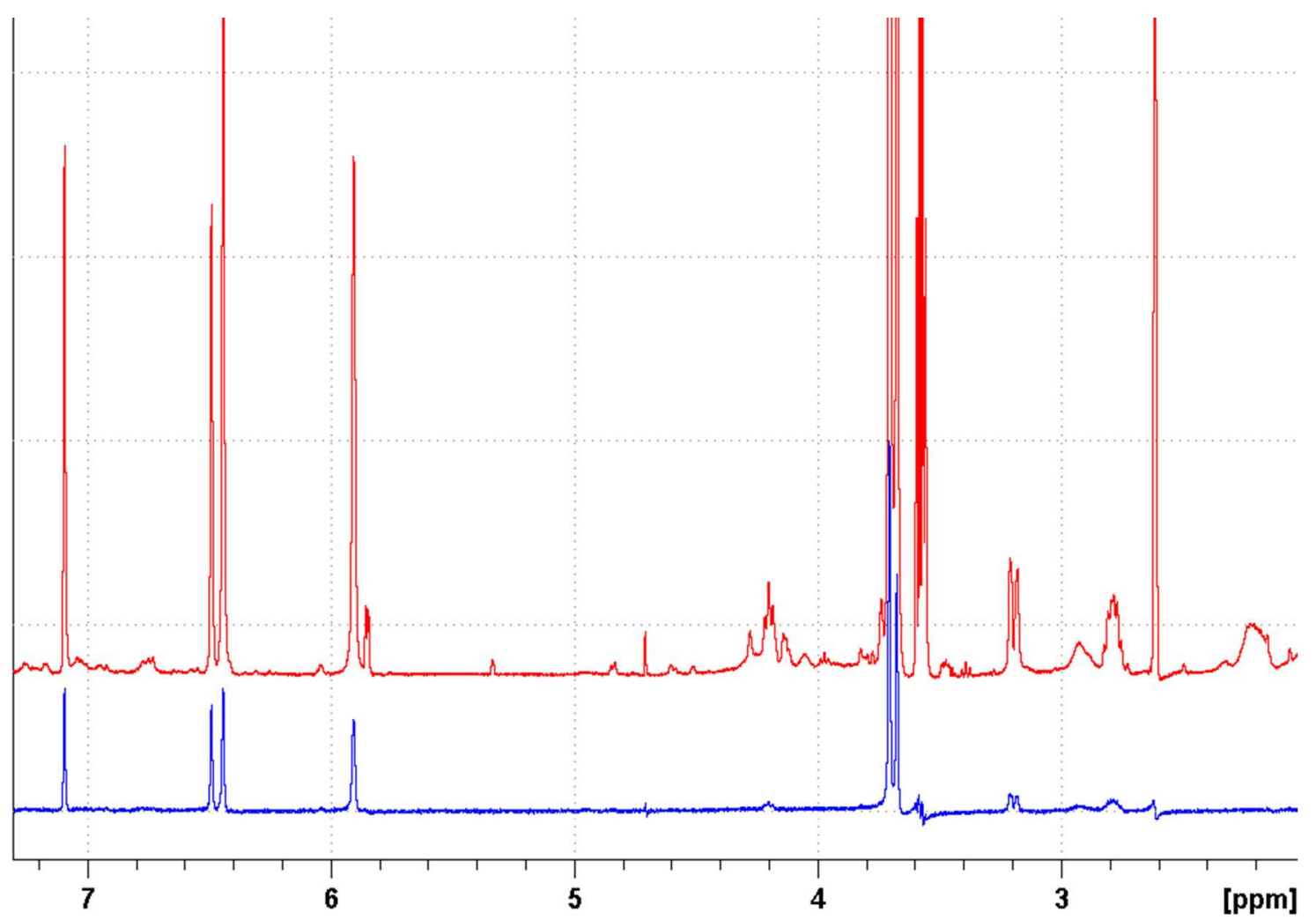

S.1. STD-NMR (600 MHz, $\left.\mathrm{D}_{2} \mathrm{O}\right)$ spectrum of compound $\mathbf{1}$. 


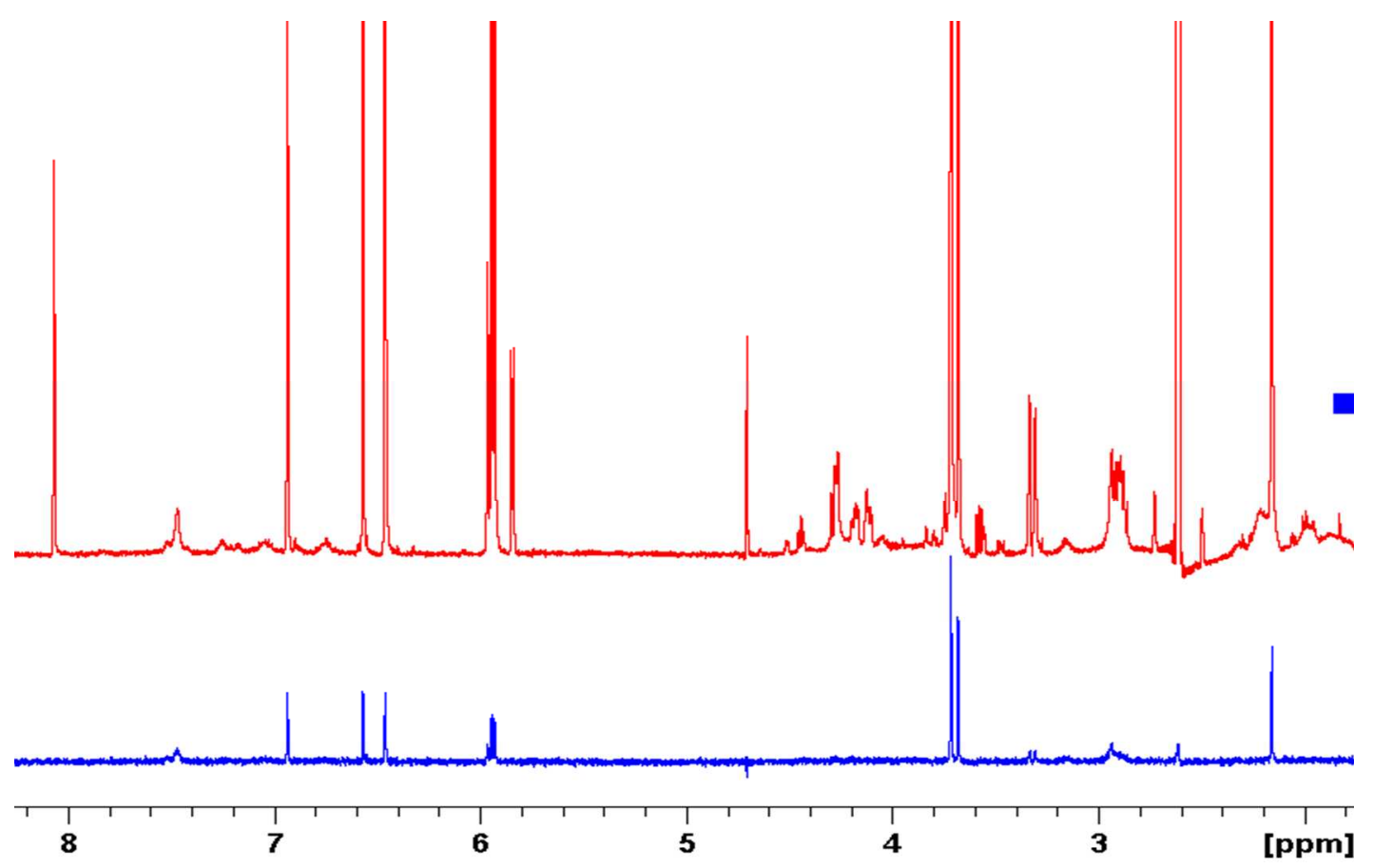

S.3. STD-NMR (600 MHz, $\left.\mathrm{D}_{2} \mathrm{O}\right)$ spectrum of compound 2.

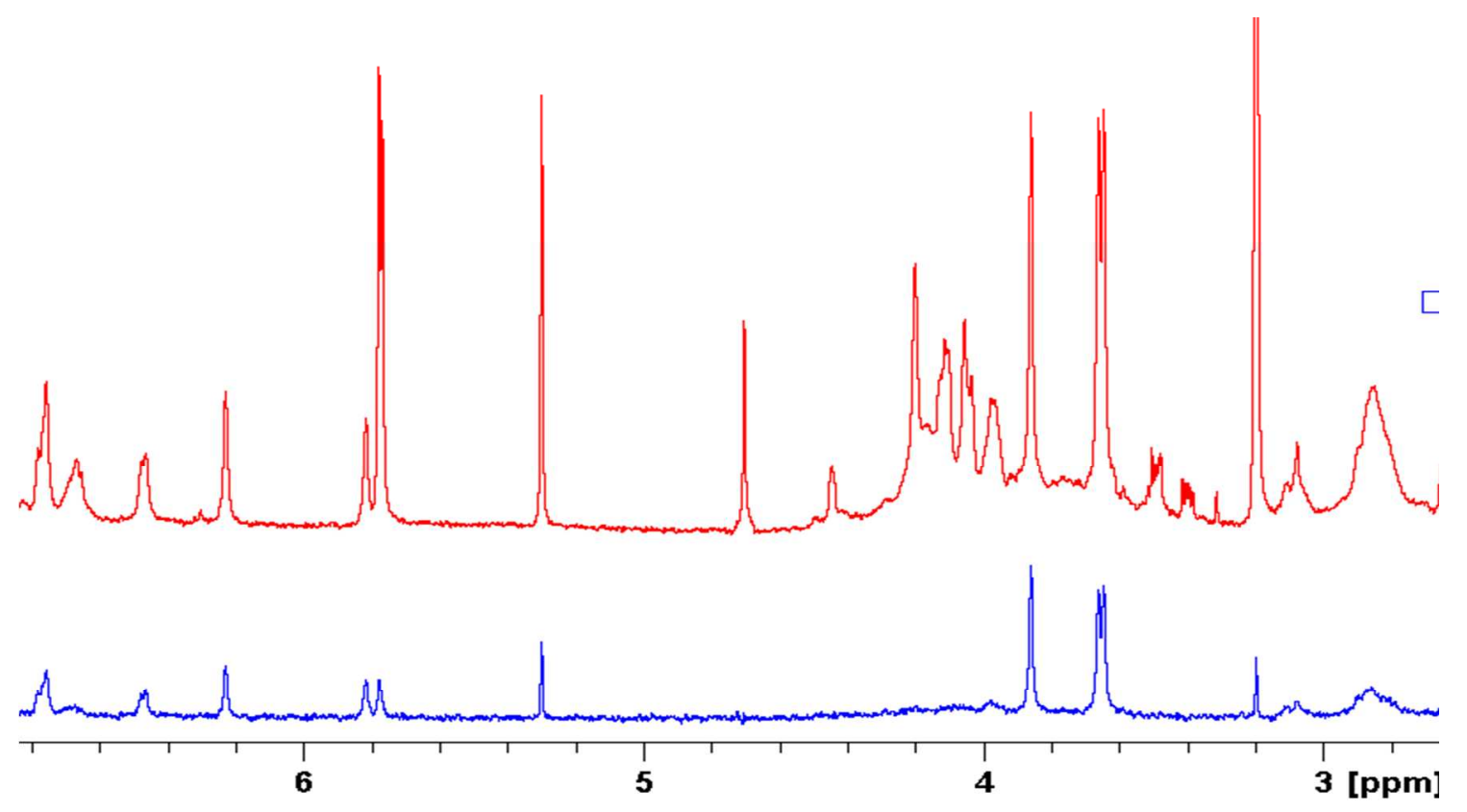

S.3. STD-NMR ( $\left.600 \mathrm{MHz}, \mathrm{D}_{2} \mathrm{O}\right)$ spectrum of compound 3. 


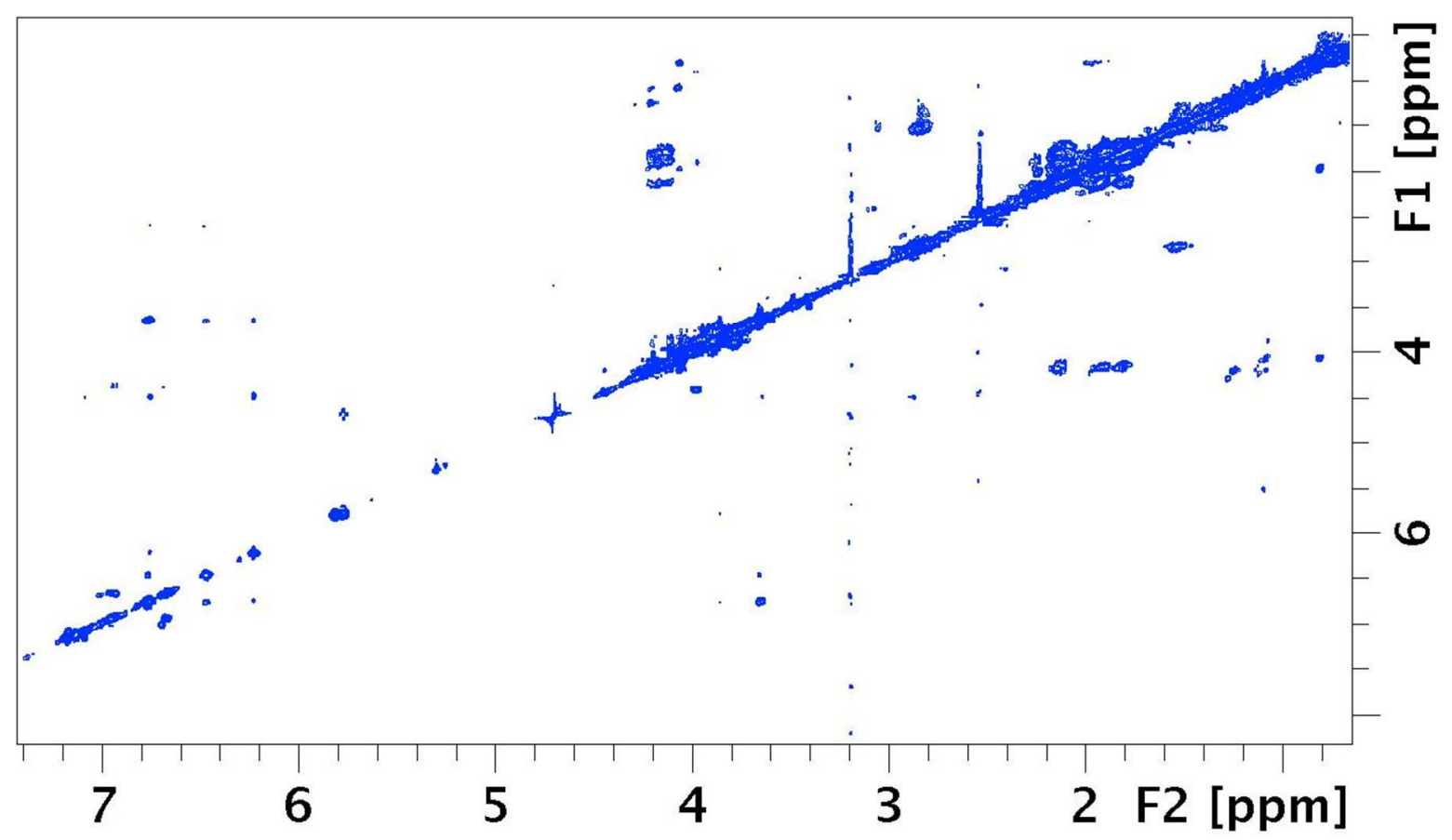

S.4. TR-NOESY (600 MHz, $\left.\mathrm{D}_{2} \mathrm{O}\right)$ spectrum of compound 3 in free state. 


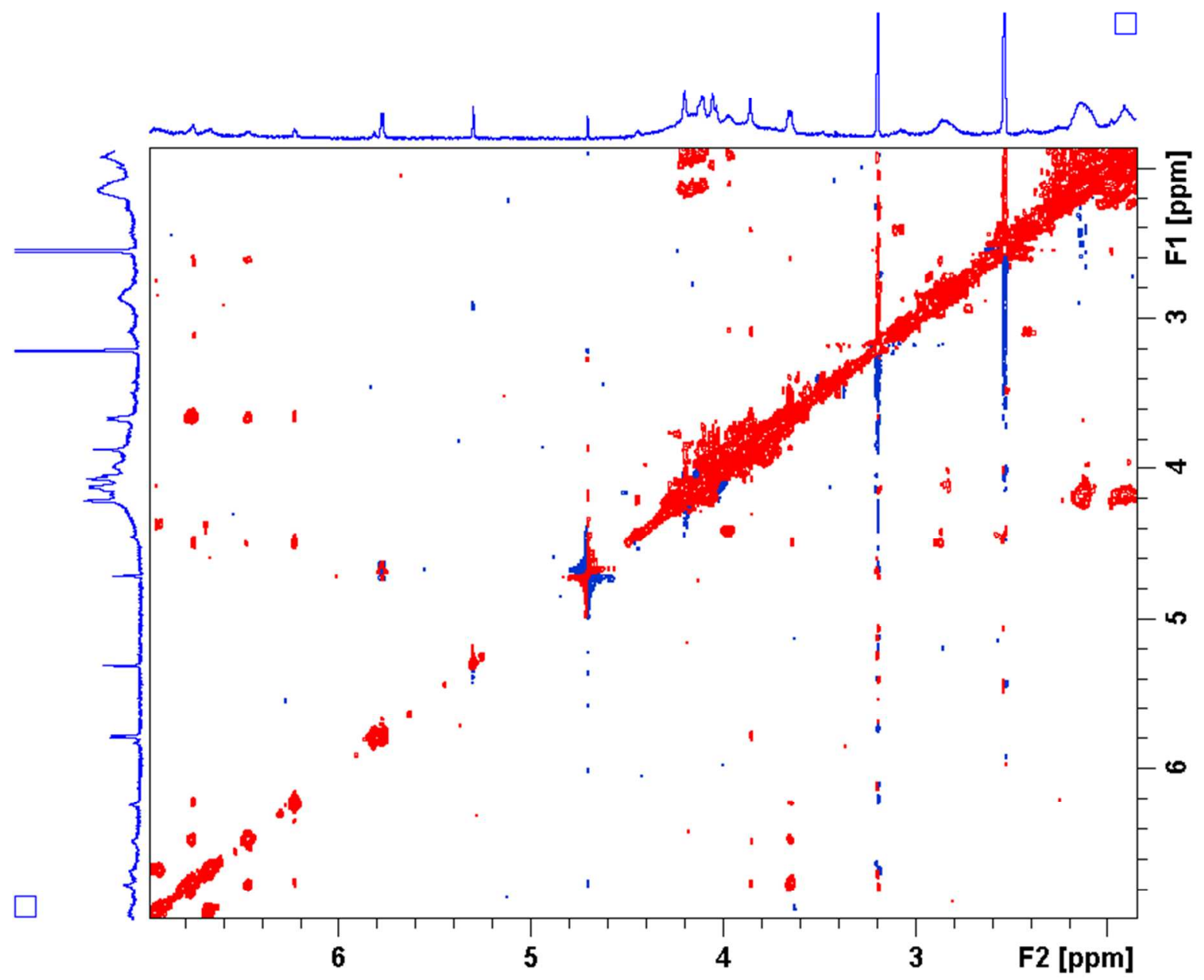

S.5. TR-NOESY $\left(600 \mathrm{MHz}, \mathrm{D}_{2} \mathrm{O}\right)$ spectrum of compound 3 in complex state with dimer of tubulin 